\section{Frühe rheumatoide Arthritis: Optimale MTX-Dosis erzielt bessere Ergebnisse}

\begin{abstract}
Mit Blick auf die Remission und die Gelenkfunktion profitieren Patienten mit früher rheumatoider Arthritis am meisten von optimal dosiertem Methotrexat. Die radiologische Progression wird dadurch aber nicht beeinflusst.
\end{abstract}

Eine ne gute Rheumatherapie führt zu besseren Resultaten als eine schlechte - das klingt zunächst nach einer Binsenwahrheit. Auf einem anderen Blatt steht freilich, ob die gute Behandlung auch jedem Rheumapatienten zuteil wird. Dass davon in der Praxis mitnichten auszugehen ist, wird durch die Ergebnisse einer Studie bestätigt, die Cécile Gaujoux-Viala von der Universitätsklinik Nîmes zusammen mit Kollegen veröffentlicht hat.

Gaujoux-Viala und ihr Team hatten die Krankheitsverläufe von 288 Patienten mit rheumatoider Arthritis (RA) im Frühstadium ausgewertet, die an der ESPOIR-Studie teilgenommen hatten. Obwohl Methotrexat (MTX) nach wie vor als Basistherapeutikum der ersten Wahl gilt, hatten nur $53 \%$ der Patienten das bewährte Immunsuppressivum wäh- rend des ersten Nachbeobachtungsjahres verordnet bekommen. Von diesen erhielten nur $26 \%$. eine optimale MTXDosis. Sie war definiert als:

- Therapiebeginn mit MTX binnen dreier Monate nach Aufnahme in die Studie; - Initialdosis $\geq 10 \mathrm{mg} /$ Woche;

- Dosis $\geq 20 \mathrm{mg} /$ Woche oder $0,3 \mathrm{mg} / \mathrm{kg} /$ Woche nach sechs Monaten, falls die Krankheitsaktivität im DAS28 einen Wert $>2,6$ erreicht (oder jede andere Dosis bei einem DAS28 < 2,6, entsprechend einer Remission).

Im Vergleich der optimal mit den nicht optimal mit MTX behandelten Patienten zeigte sich ein deutlicher Vorteil für Erstere. $27 \%$ der optimal Behandelten befanden sich nach einem Jahr in Remission, bei den nicht optimal Therapierten waren es $8 \%$. Eine normale Gelenkfunktion hatten $74 \%$ versus $51 \%$. Und $25 \%$ versus $10 \%$ wurden in Kombination mit anderen Basistherapeutika behandelt. An den besseren Ergebnissen unter korrekter MTX-Dosierung änderte sich auch im zweiten Jahr des Follow-up nichts. Vorteile bezüglich der radiologischen Progression waren allerdings nicht zu erkennen. Nach einem Jahr waren bei $69 \%$ versus $71 \%$ der Patienten mit optimaler beziehungsweise suboptimaler MTX-Behandlung keine Zeichen eines raschen Fortschreitens der Krankheit erkennbar. Nach zwei Jahren waren es $75 \%$ respektive $81 \%$.

Fazit: Die Ergebnisse der Studie zeigen nach Meinung der Autoren, dass Ärzte verstärkt darauf achten sollten, bei früher RA das Basistherapeutikum MTX besser einzusetzen, also: innerhalb von drei Monaten nach der Diagnose und optimal dosiert.

Dr. Robert Bublak

Gaujoux-Viala C et al. Optimal methotrexate dose is associated with better clinical outcomes than non-optimal dose in daily practice: results from the ESPOIR early arthritis cohort. Ann Rheum Dis 2017; http://dx.doi.org/10.1136/ annrheumdis-2017-211268

kant häufiger über Benommenheit, Müdigkeit, Schwierigkeiten beim Denken und Sehstörungen als unter Placebo beziehungsweise aktiven Therapien.

Der Off-Label-Einsatz von Gabapentin und Pregabalin bei chronischer unspezifischer Lumbalgie wird durch die wenigen Studien in dieser Indikation nicht gestützt. Dies hat eine Metaanalyse der vorhandenen Daten ergeben

B islang wurden gemäß Harsha Shanthanna et al. von der McMaster-Universität in Hamilton/USA nur acht randomisierte kontrollierte Studien zum Einsatz von Gabapentinoiden bei mehr als drei Monate anhaltenden Kreuzschmerzen veröffentlicht. Die gemeinsame Auswertung der drei Studien zu Gabapentin mit insgesamt 185 Patienten ergab im Placebovergleich lediglich eine minimale Schmerzreduktion, die mittlere Differenz auf einer Skala von 0-10 betrug nur 0,22 Einheiten. Die Qualität der Evidenz wurde mit dem GRADESystem als „sehr niedrig“ eingeschätzt.
Für Pregabalin gab es - Stand Ende 2016 - nur Studien, in denen der Wirkstoff, allein oder als Ergänzung einer Schmerztherapie, anderen Therapien gegenübergestellt wurde. Die Pregabalinmonotherapie schnitt in drei Studien mit insgesamt 332 Patienten weniger gut ab als die Vergleichssubstanzen (Ami triptylin, Celebrex oder Tramadol/Paracetamol): Der mittlere Unterschied zugunsten der anderen Wirkstoffe betrug 0,42 Einheiten bei sehr geringer Evidenz.

Gravierende Nebenwirkungen traten unter den Gabapentinoiden nicht auf. Die Patienten berichteten aber signifi-
Fazit: Wegen des unbewiesenen Nutzens raten die Autoren zur Vorsicht bei der Gabe von Gabapentinoiden gegen chronische Rückenschmerzen.

Dr. Beate Schumacher

Shanthanna $\mathrm{H}$ et al. Benefits and safety of gabapentinoids in chronic low back pain: A systematic review and meta-analysis of randomized controlled trials. PLoS Med 14(8); https://doi. org/10.1371/journal.pmed.1002369 